\title{
Scaling laws from theory-based modeling for different regimes in DEMO fusion reactor
}

\author{
F. Palermo ${ }^{1}$, E. Fable ${ }^{1}$, C. Angioni ${ }^{1}$, M. Siccinio ${ }^{1,2}$, \\ H. Zohm ${ }^{1}$ \\ ${ }^{1}$ Max Planck Institut fur Plasmaphysik, Boltzmannstr. 2, 85748 Garching bei \\ Munchen, Germany \\ ${ }^{2}$ EUROfusion Consortium, Boltzmannstr. 2, 85748 Garching bei Munchen, \\ Germany \\ E-mail: francesco.palermo@ipp.mpg.de
}

\begin{abstract}
In the European Union strategy, DEMO is the single step between ITER and a fusion power plant. The current state of physical knowledge and technological limits doesn't yet well allow us to identify the best combination of solutions to decide the architecture of this new tokamak. To this purpose, an important element of tokamak research finalized to the prediction, the interpretation and the planning of experiments is represented by the "integrated modeling". In this work we use the integrated modeling ASTRA tool coupled with the TGLF transport model in order to investigate scenarios of interest for DEMO. We obtain scaling laws which describe and serve as a guide-line for the pre-conceptual design of the machine. In particular, by means of scaling laws we investigate the performances of the reactor in terms of fusion power, confinement time $H$-factor and fusion power multiplication factor $Q$, in different regimes such as $H$-mode, $I$-mode and $L$-mode. In this way we provide important key points for the establishment of the best physical and technological solutions for DEMO scenarios.
\end{abstract}

fusion reactors, tokamak, $H$-mode, $I$-mode, $L$-mode, reactor scenario 


\section{Introduction}

Among the toroidal magnetic confinement devices, tokamaks represent the most exploited machines to achieve practical fusion energy. The preference for tokamak machines is due to their simplicity and to the quality of the results obtained. Thus, in the next future, the perspective of fusion reactors are mainly based on the generation of tokamak devices such as ITER $[1,2,3]$. However, it is planned that ITER will generate only ten times $(Q \approx 10)$ the power needed to produce and to heat the plasma [2]. This amount of energy is not enough for a power plant. Thus, in parallel to the physics investigations and to the engineering development of ITER, an accompanying fusion technology program dubbed DEMO (DEMOnstration Fusion Power Plant) is going to be developed $[4,5]$. The DEMO machine is the objective of the EUROfusion Power Plant Physics Technology (PPPT) Work Programme [6, 7, 8]. This programme should determine a transition between physics experiments and commercial fusion power plants. It is predicted that the DEMO size will be characterized by a major radius $R \approx 9 \mathrm{~m}$ and the production of net electrical power will be around 500 MW. The confinement properties of the resulting plasma can be evaluated by modeling the plasma heat transport with numerical simulations. To this purpose, an important element of tokamak research aimed at the prediction, the interpretation and the planning of experiments is represented by "integrated transport modeling". This set of increasingly validated simulation tools merges the scientific knowledge accumulated in plasma fusion research. An example of integrated modeling is the ASTRA tool [9] that is composed by a suite of numerical codes for the predictive/interpretative simulation of a full tokamak discharge. It integrates, in a modular structure, a $1 D$ transport solver with a general $2 D$ magnetic equilibrium solver, heat, particle, momentum and impurities transport models, as well as all related sources.

The design of a machine and in particular of DEMO, requires a careful iteration between a transport code, like ASTRA, and specific codes devoted to calculate in detail relevant aspects of physics such as turbulent transport. In the present work ASTRA has been coupled with the TGLF code able to compute fluxes for heat and particle transport produced by turbulence [31]. The code calculates the solution of fluid moments with a kinetic closure fitted to match kinetic responses computed with linear gyrokinetic codes.

One of the possible use of this package is the derivation of scaling laws obtained via parameter scans. Scaling laws are very important for different reasons. They can be used as a reference for the comparison of several configurations and for the prediction of the corresponding physical parameters of future machines. Great attention has been given to improve these laws in particular concerning the energy confinement time. Scaling laws have been derived for the prediction of ITER performance [2], but not enough studies have been done for DEMO. Thus, here we used the ASTRA and TGLF codes to investigate and to derive scaling laws for fusion power $P_{\text {fus }}$ and $H$ factor for a tokamak plasma around typical sizes and geometrical parameters as for DEMO. In this way we would like to find a parametric evaluation of the DEMO performance able to encompass different plasma confinement regimes. We perform several scans in different parameters in a range of values finalized to study several important regimes such as high confinement ( $H$-mode) [11] and improved confinement (I-mode) [12]. In particular, the main parameters that we consider in our scans are, for ions and electrons, the temperature $T_{0}$ and the density $n_{0}$ at the pedestal top. We vary these two parameters in a large range of values in order to cover physical conditions that allow us to distinguish between different regimes. $H$-mode is the reference operating scenario in future reactors and in particular in ITER. In the latter, the structure of the pedestal is thought to determine good plasma performance and to induce instabilities near the edge (ELMs), that transiently increase the heat load on the divertor [13]. Although there is confidence that this regime can be accessed with sufficient performance, the ability to produce $H$-mode plasmas with small ELMs is an important step towards extending the lifetime of the divertor target plates in ITER [14]. The problem will be more important in DEMO having a severe impact on machine availability. For these reasons, the investigation of DEMO performances in several regimes, and in particular in the "ELM-free" ones, can be very useful. Between the potential attractiveness ELM-free fusion scenarios, I-mode received increasing attention in recent years. Thus, the general scaling laws obtained in this study to describe a large range 
of scenarios for DEMO, have been applied to compare $H$-mode performance with that one of a representative ELM-free regime such as the $I$-mode $[15,16]$. This latter is characterized by a pedestal temperature similar to the $H$-mode, without a density pedestal. We emphasize that the $I$-mode has the advantage over the ELMy $H$-mode of eliminating the transient heat flux pulses to divertor $[18,16,19]$. An important aspect that concerns the $I$-mode is the understanding of the threshold condition $L-I$ and $I-H$ to maintain the $I$-mode in a stationary phase. To this purpose, the magnetic field plays an important role to maintain the pedestal conditions of $I$-mode avoiding the $I-H$ transition [17]. We also investigate the $L$-mode regime in order to give a complete picture of the value of the principal parameters involved in a power plant based on DEMO. The present paper is organized as follows. In Sec. II, we briefly recall the set of equations used in our model and we present the methodology adopted to make the different scans. In Sec. III, we discuss the reference parameters used to define the geometry of DEMO and we report and interpret results of the performed simulations. Finally in Sec. IV, we present the conclusions and the implications of this work.

\section{Numerical model and physical approach}

\subsection{ASTRA code}

The ASTRA code is a highly flexible tool dedicated to integrated simulations for the solution of a variety of transport problems in magnetically confined plasmas. For this property and effectiveness ASTRA represents a very popular transport simulation tool in the fusion community. The code has been presented and discussed in several works $[20,21,22]$, therefore in this section we briefly summarize the main features used in the code. Detailed derivation and discussions of the model can be found in Ref. [9]. ASTRA has a modular organization supported by the method of the code creation. Each block necessary for simulations is connected to other blocks through the ASTRA environment providing uniform control and exchange capability. The basic set of equations in the ASTRA code includes expressions for the electron density $n_{e}$, electron temperature $T_{e}$, ion temperature $T_{i}$ and poloidal flux $\phi$. Independently of the origin of the equations used for the representation of the fluxes, the schematic structure of ASTRA assumes a transport matrix that connects the flux of energy and the source terms for electrons $\mathcal{P}_{e}$ and ions $\mathcal{P}_{i}$ respectively. In the following, we indicate with $\mathcal{P}$ the general source of heat power density (measured in $\mathrm{MW} / \mathrm{m}^{3}$ ) and we indicate with $P$ the general power integrated on the tokamak volume. The source terms used in the present work are:

$\mathcal{P}_{e}=\mathcal{P}_{\alpha, e}+\mathcal{P}_{\text {Ohm }}-\mathcal{P}_{e i}-\mathcal{P}_{e, \text { rad }}+\mathcal{P}_{e, a u x}$

$\mathcal{P}_{i}=\mathcal{P}_{\alpha, i}+\mathcal{P}_{\text {ei }}+\mathcal{P}_{i, a u x}$

In Eq. 1 the density power of Ohmic heating $\mathcal{P}_{\text {Ohm }}=$ $\eta j^{2}$ is linked to the plasma resistivity and to the density current $j$. The two terms $\mathcal{P}_{\alpha, e}$ and $\mathcal{P}_{\alpha, i}$ are the fractions of born $\alpha$-particle power $\mathcal{P}_{\alpha}$ absorbed by electrons and ion respectively. The $\alpha$-particle power $\mathcal{P}_{\alpha}$ is related to the fusion power $\mathcal{P}_{\text {fus }}$ through the well-known relation $\mathcal{P}_{\alpha}=f_{\text {coup }} \mathcal{P}_{\text {fus }}$ with $f_{\text {coup }}=20 \%$ [23]. For reactor scenario, $\mathcal{P}_{\alpha}$ represents the most important contribution between the different powers in Eqs. 1 and 2. Concerning the $\alpha$-particle heating per unit volume, ASTRA uses the classical cross section expression reported, for example, in Ref. [23]. For the range of parameter values considered in this work, the fractions $P_{\alpha, i}$ and $P_{\alpha, e}$, absorbed by ion and electrons respectively, exhibit the following trend:

$\frac{\mathcal{P}_{\alpha, i}}{\mathcal{P}_{\alpha}} \propto T_{i}^{0.6} \quad \mathcal{P}_{\alpha, e}=\mathcal{P}_{\alpha}-\mathcal{P}_{\alpha, i}$

The exchange of energy linked to the term $\mathcal{P}_{\text {ei }}$ between ions and electrons is regulated by the Coulomb interaction. The power transferred between ions and electrons is $\mathcal{P}_{e i}=3 / 2 n_{i}\left(T_{e}-T_{i}\right) / t_{e q}$ where $t_{e q}$ is the equipartition time [9]. This time generally is large enough with respect to other phenomena that allows us to distinguish between electron $T_{e}$ and ion temperature $T_{i}$. In DEMO, $T_{e}$ and $T_{i}$ can be different in the core (usually $T_{e}$ larger than $T_{i}$ ), but are quite similar towards the edge region. Powers of auxiliary heating is given by $\mathcal{P}_{\text {aux }}=\mathcal{P}_{\text {e,aux }}+\mathcal{P}_{i, a u x}$ with $\mathcal{P}_{\text {e,aux }}=\mathcal{P}_{\text {i,aux }}$. Radiation power $\mathcal{P}_{\text {rad }} \approx$ $\mathcal{P}_{e, \text { rad }}$ is decomposed in bremsstrahlung radiation of electrons due to the interaction with ions, electron synchrotron radiation particularly important at high temperatures and radiation due to the impurity of the plasma. For the bremsstrahlung radiation we used the classical formula that can be found for example in Ref. [23] and for the synchrotron radiation we used the well-know Trubnikov scaling [24]. Concerning the radiation line emission, for the Xenon impurity we used the expressions reported in Ref. [25]. Usually, a tokamak plasma contains a variety of different species. These might be hydrogen isotopes, helium, or other impurities. Each of them can be characterized by its own density and temperature. Non-Maxwellian populations of the main plasma components can also be treated as independent species. The system of onedimensional transport equations is coupled to the $2 D$ equilibrium solver SPIDER [29, 21]. In the present application, the boundary conditions of the plasma are described via the three moments parameters: plasma elongation $\kappa$, triangularity $\delta$ and minor radius $a$. Because of this combination of $1 D$ and $2 D$ equations, 
transport codes of this type are often referred to as $1.5 \mathrm{D}$ code. With appropriate initial conditions and boundary conditions (see Sec. 3), we can determine the time evolution of the radial distributions of the electron density $n_{e}$, ion and electron temperature $T_{i}$ and $T_{e}$ respectively and current density. As mentioned, the relation between source and flux is generated by a matrix that includes the heat conductivity coefficient $\chi$ and the convection of particles $C$. The elements of the transport matrix are divided in two components linked to the neoclassical and to the turbulent transport respectively. In this work, the first part is calculated in ASTRA with the module NCLASS [30] and the second part is calculated by means of TGLF $[31,32,33]$.

\subsection{TGLF code}

The TGLF code is well implemented in ASTRA and different interfaces between ASTRA and TGLF are available, for both serial and parallel computations. TGLF is a code based on first principles with a more comprehensive physics than the models previously developed. In particular, the trapped and passing particle descriptions have been majorly upgraded with respect to its predecessor GLF23 [31, 34]. Therefore, the code is suitable to analyze the physics at the basis of the investigated scenarios including principal instabilities due to ion temperature gradient (ITG), electron temperature gradient (ETG), trapped electron mode (TEM) and zonal flow mixing saturation process able to reproduce the nonlinear $k_{y}$-spectrum of the multiscale saturated potential [35]. The code also takes into account toroidal geometry of the plasma and electromagnetic effects such as fluctuations that can affect the magnetic field. Thus, TGLF is able to compute turbulence-driven fluxes for heat and particle transport and for its characteristics it is particularly suitable to investigate DEMO scenarios. The reliability of TGLF has been improved via comparisons with gyrokinetic simulations. In these years the TGLF model has been fitted to a large database of gyrokinetic simulations derived from GYRO [36]. An important area where TGLF shows good agreement with simulations is the plasma shaping. In fact one of the goals in developing TGLF was to incorporate Miller shaped geometry and to test the model against GYRO simulations for a variety of plasma elongations and triangularities [37]. A successful comparison between the TGLF and experimental results has been obtained by using several discharges from DIII-D [38], JET, TFTR [33] and ASDEX Upgrade [39, 40]. In particular, JET discharges in the presence of high triangularity, high $\beta_{N}$ and high toroidal rotation have been simulated with TGLF showing a good agreement between experiments and code results and giving some indication for the improvement of transport models
[41]. Recent results from DIII-D [42, 43] also suggest that it is possible to reproduce temperature profiles in high- $\beta$ steady-state scenarios with TGLF. From the point of view of TGLF validation in different regimes, we observe that significant progress has been made on $L$ - and $H$-mode $[26,28]$. Recently, the attention on the TGLF validation moves also towards other regimes such as the $I$-mode [27]. To this purpose, the present work gives further motivation to the application and validation of the TGLF model to the $I$-mode regime.

\subsection{Rationale}

In this work we performed several scans on different parameters in order to investigate relevant regimes for DEMO. As we have mentioned in the introduction, the goal of the work was to obtain scaling laws for fusion power and $H$-factor parameters. Scaling laws should reflect the equations which govern the plasma confinement. Many efforts have been made in order to obtain expressions in terms of dimensionless parameters such as $\beta$ value, collisionality, normalized gyro radius. However, it can be useful to present scaling laws in terms of more practical parameters which can be controlled externally. In the following we present $P_{\text {fus }}$ and energy confinement time via the $H$-factor as a function of geometrical parameters such as major radius $R$ and aspect ratio $A$, external parameters such as magnetic field $B$, current $I_{p}$ (or safety factor $q$ ), auxiliary and radiation power and pedestal parameters such as density and temperature (or equivalently pressure $p$ ). We focus our attention on temperature and density at the pedestal top and we note that the edge pedestal represents the most critical region that must be predicted/described in the integrated modeling. Due to the incomplete knowledge of the pedestal physics the coupling between core and pedestal region is problematic and would require a careful dedicated study. By means of our scans, we related the physics of the core with the pedestal physics that we modeled through two fundamental parameters that are temperature $T_{0}$ and density $n_{0}$ at the pedestal top. We observe that density $n_{0}$ also is an external parameter, whose value can be regulated in the experiments. The temperature at the pedestal top $T_{0}$ is not independent, but can be expressed as a function of the other quantities on the basis of the models which will be adopted by the users. Thereby, in this work the temperature $T_{0}$ is treated as a free parameter in order to increase the applicability of the proposed scaling laws. The results have been then described by means of regressions delivering scaling laws for the fusion power and $H$-factor. The impact of a concatenated variation of multiple parameters can be investigated by the use of these scaling laws. Thus, in our scans each parameter has been varied 
by maintaining the other ones fixed. It is important to emphasize that general scaling laws valid for all experiments cannot be expected. For this reason, it is very important to identify the limits and the region of validity of the different expressions. Afterward, we will discuss the physical motivations about the validity limits of the obtained laws.

\section{Results}

The principal values of parameters, that in the following we consider as a reference, are summarized in table 1 . In particular we choose a major radius $R=9$ $\mathrm{m}$ and a minor radius $a=2.9 \mathrm{~m}$ that corresponds to have a tokamak with a volume $V=2465 \mathrm{~m}^{3}$ with an aspect ratio $A=R / a=3.1$. For magnetic surface geometry we fix tokamak elongation at $\kappa=1.7$ and triangularity at $\delta=0.35$ for all the simulations. We consider a Deuterium-Tritium (DT) plasma with a Helium fraction of $8.7 \%$ for the nominal case. It is important to note that the Helium concentration depends on the fusion power and could be calculated in a self-consistent way. However, the final balance of Helium quantity also depends on the transport and on the external conditions like pumping. A dedicated study on this balance is not part of this work and consequently we consider the helium fraction basically as a fix number. Instead, the Xenon concentration is regulated on the basis of the power value flowing through the plasma boundary. A study of this quantity is included in an independent scan performed on the radiation power $P_{\text {rad }}$. The impact of impurities on the turbulent transport is not taken into account and impurities only appear as a dilution factor for the rate of fusion reactions in the plasma. In this study we focus our attention on baseline mode regimes such as standard $\mathrm{H}$-mode scenario like those considered in the international database used for the scaling IPB98y [2]. As explained, in this work we modeled several regimes by fixing the physical condition such as temperature and density values at the pedestal.

The crossing conditions between core and pedestal are chosen at $r / a=0.87$. In the external plasma region $r / a>0.87$ the profiles are fixed and the transport coefficients are irrelevant for the sake of the present investigation. The pedestal position is around $r / a \approx 0.92$ that corresponds to have a pedestal width $\Delta r \approx 23 \mathrm{~cm}$. The current is fixed at $I_{p}=$ 17.75 MA that is considered an acceptable value from the technological point of view of coil stress. The safety factor is chosen with a value $q_{95} \approx 4$ and with a minimum value equal to 1 in the core region as if there was a continuum sawtooth activity. This is because we consider the less optimistic situation from the point of view of the confinement. We will extensively discuss
Table 1. Nominal values of parameters for DEMO

\begin{tabular}{ll}
\hline Parameters & Values \\
\hline plasma current & $I_{p}=17.75 \mathrm{MA}$ \\
Nominal toroidal field & $B=5.8 \mathrm{~T}$ \\
Major radius & $R=9.01 \mathrm{~m}$ \\
Minor radius & $a=2.9 \mathrm{~m}$ \\
Aspect ratio & $A=R / a=3.1$ \\
Elongation & $\kappa=1.7$ \\
Triangularity & $\delta=0.35$ \\
\hline
\end{tabular}

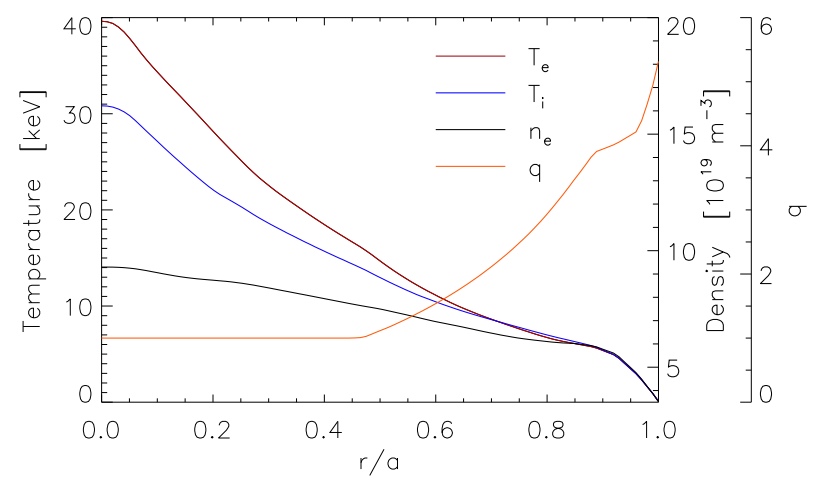

Figure 1. Equilibrium profiles of density, safety factor $q$, electron $T_{e}$ and ion $T_{i}$ temperature respectively, for the chosen nominal case.

the confinement dependence on the safety factor profile in a future work. In Fig. 1 we can find the principal equilibrium kinetic profiles. We observe in particular that at $r / a=0.5$ the temperature is around $T_{i}=13 \mathrm{keV}$. This corresponds to have a Larmor radius $\rho_{L}=m v_{\perp} /(e B) \approx 3 \cdot 10^{-3} \mathrm{~m}$ that corresponds to $\rho_{*}=\rho_{L} / a \approx 10^{-3}$. The small value of $\rho_{*}$ implies that the global effects are negligible and this justifies the local approach to the problem adopted in our model [44]. Results of the nominal case are obtained with an auxiliary power $P_{a u x}=48.2 \mathrm{MW}$ and a radiation power $P_{\text {rad }}=133.5 \mathrm{MW}$. The definition used for the confinement time is:

$\tau_{E}=\frac{W_{e}+W_{i}}{P_{a u x}+P_{\alpha}+P_{\text {Ohm }}}$

where $P_{\text {Ohm }} \ll P_{\text {aux }}+P_{\alpha}$ and where $W_{e}$ and $W_{i}$ are the thermal energy for ions and electrons respectively. For the nominal case, the confinement time is $\tau_{E} \approx$ 3 s. The physical mechanisms that determine the confinement time $\tau_{E}$ are related to the radial fluxes and to ASTRA transport equations via electron and ion heat conductivity $\chi_{e}$ and $\chi_{i}$ respectively. In Fig. 2 we show the radial profiles of these two parameters putting in evidence that $\chi_{i}$ is around two times larger than $\chi_{e}$. By observing the equilibrium profiles on Fig. 1 and 


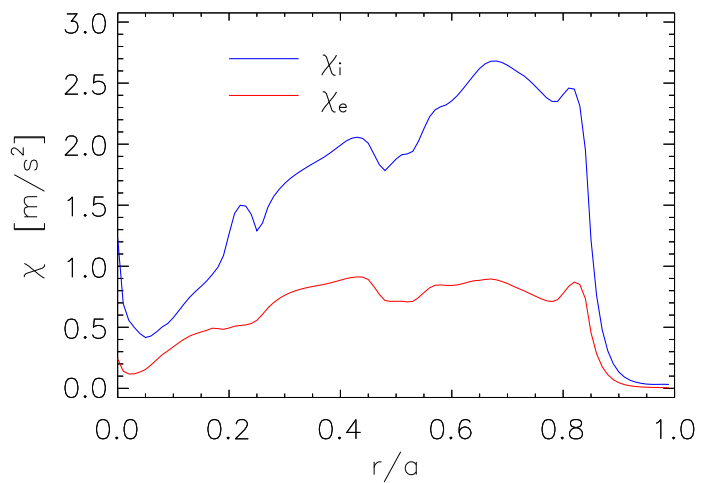

Figure 2. Radial profiles of electron $\chi_{e}$ and ion $\chi_{i}$ heat conductivity for the nominal case.

the values of $\chi_{i}$ and $\chi_{e}$ on Fig. 2, we deduce that the heating flux for ions $Q_{i}=-n_{i} \chi_{i} \nabla T_{i}$ is larger than that one of electrons $Q_{e}=-n_{e} \chi_{e} \nabla T_{e}$. Consequently, the principal instability in our simulations is determined by the ion temperature gradient (ITG). As we have verified, this characteristics is common for all the simulations performed in our scans and therefore for the different regimes ( $H$-, $I$ - and $L$-mode) investigated in the work. Moreover, $\tau_{E}$ is strongly influenced by the choice of the pedestal values, as these make an important part of the stored energy. We observe that, for the reference case, the stored energy fraction in the core is around $50 \%$ of the stored total energy.

\subsection{Scan in $T_{0}$ and $n_{0}$}

We investigate the effects of temperature $T_{0}$ and density $n_{0}$ at the pedestal top on the performance of DEMO. For the temperature we consider as a reference value $T_{0}=5.5 \mathrm{keV}$ (see Ref. [51]). Thus, we performed a scan in which we vary the temperature in the range $3.5 \mathrm{keV} \leq T_{0} \leq 6.2 \mathrm{keV}$ that covers typical values expected for $H$ - and $I$-mode in DEMO. Due to several constraints, the density is a parameter more critical than the temperature. About density, the most important constraint is represented by the Greenwald limit $n_{G w}$ [45]. This is an empirical law that, for a given plasma current, determines the maximum achievable linear-averaged density in the plasma:

$n_{G w}=\frac{I_{p}}{\pi a^{2}}$

For our nominal case with $I_{p}=17.75 \mathrm{MA}$ and $a=3 \mathrm{~m}$ we have $n_{G w}=6.2 \cdot 10^{19} \mathrm{~m}^{-3}$. For the density scan we choose the following range $0.8 n_{G w} \leq n_{0} \leq 0.9 n_{G w}$. We note the scan never overtakes the Greenwald limit. However, it is important to note that several effects have been discovered that allow one to work in $H$ mode at densities close to and even higher than the Greenwald limit. For example, the triangularity can play an important role in determining the density limit at which the stability drops [46]. This could be connected with the variation of the minor radius $a$ with the poloidal direction and with the plasma shape that determines the pedestal stability. Other factors that can influence the Greenwald limit are the injection of pellet [47]. Moreover, discharges on DIII-D [48] and on ASDEX Upgrade [49] demonstrate that the possibility to exceed the Greenwald limit is related to the plasma edge and to the central peaking.

\subsection{Scan in $P_{a u x}$ and $P_{\text {rad }}$}

We perform a scan in $P_{\text {rad }}$ and $P_{a u x}$. In particular we consider the following range: $0 \mathrm{MW} \leq P_{\text {aux }} \leq$ $100 \mathrm{MW}$ and $30 \mathrm{MW} \leq P_{\text {rad }} \leq 235 \mathrm{MW}$. In the balance of power in the tokamak plasma the loss and the auxiliary powers play a fundamental role. The losses of energy in a plasma are due to the radiation, transport of heating and loss of particles. In this work, we do not solve self-consistently transport equations for the impurities, but we limit ourselves to mimic their presence by adjusting the total core radiation value. In our model, the radiated power can be decomposed in bremsstrahlung of electrons due to the interaction with ions and electron cyclotron radiation that becomes important at high temperatures. Auxiliary power for plasma heating $P_{a u x}$ is distributed in equal amounts between electrons and ions, while $P_{\text {rad }}$ is due principally to electrons. Moreover, as we show in Fig. 3, auxiliary power $\mathcal{P}_{\text {aux }}$ together with $\mathcal{P}_{\alpha}$ involves all the central region of tokamak, while $\mathcal{P}_{\text {rad }}$ principally involves the edge region.

\subsection{Scan in $R, B, I_{p}$ and $A$}

The investigation of parameters such as aspect ratio $A$ and consequently major $R$ and minor radius $a$, magnetic field $B$ and current $I_{p}$ is crucial for the design of a fusion reactor. These parameters are involved in several physical and technological problems and constraints. For example the maximum current limit of a tokamak depends on many different factors such as coil stress. Other limits are due to physical reasons and can be derived even from simple models or calculated numerically. For example all the mentioned four parameters are involved in the following relation:

$q_{e d}=\frac{2 \pi}{\mu_{0}} \frac{R B}{I_{p} A^{2}}$

where $q_{e d}$ is the safety factor at the edge of the tokamak in circular poloidal shape. This formula determines the so called Kruskal-Shafranov limit that involves the edge safety factor directly related to the plasma 


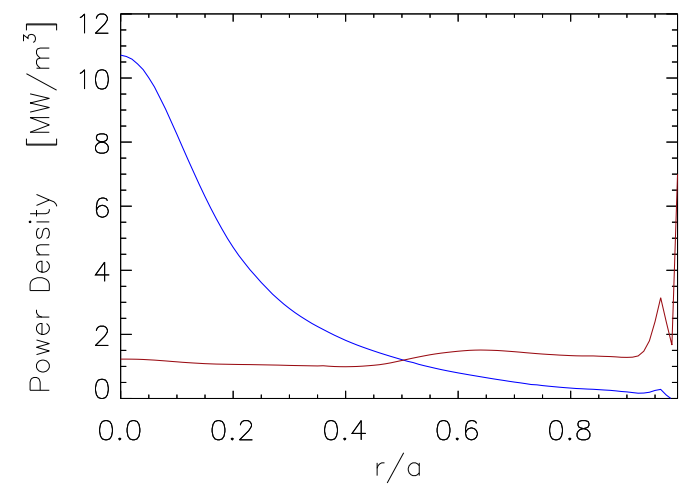

Figure 3. Radial distribution of $\mathcal{P}_{\alpha}+\mathcal{P}_{\text {aux }}$ (blue line) compared with $\mathcal{P}_{\text {rad }}$ (red line). The former is concentrated in the core of the tokamak, while the latter in particular concerns the edge.

current. Stable operations require sufficiently high values of $q$ at the edge with a consequent limitation of the maximum plasma current. Elevated values of current can generate unstable kink modes that lead to a strong and fast deformation of the plasma boundary and the plasma disruption. However, several effects can increase the current limit. For a given aspect ratio and magnetic field, higher plasma currents can be obtained by modifying the circular poloidal shape of magnetic field. In particular by changing the combined effect of the plasma elongation and triangularity it is possible to obtain a D-shaped cross-section, which is used in all modern tokamaks, that allows us to reach higher plasma current limit. We perform scans in the following range $16 \mathrm{MA} \leq I_{p} \leq 19 \mathrm{MA}$ by maintaining the other parameter values equal to those of the nominal case. From Eq. 6 we deduce that this is equivalent to do a scan in $q$. Moreover, we perform scans in $B$ and $R$ and $A$ by considering $5.8 \mathrm{~T} \leq B \leq$ $6.2 \mathrm{~T}, 8.5 \mathrm{~m} \leq R \leq 9 \mathrm{~m}$ and $2.9 \leq A \leq 3.1$ respectively.

\subsection{Scaling laws and physical interpretation}

Here, we present the results obtained from the scans described in the previous subsections. In Fig. 4, for the case $B=5.8 \mathrm{~T}$ and $R=9 \mathrm{~m}$, we have the fusion power $P_{f u s}$ as a function of the temperature respectively for $n_{0} / n_{G w}=0.9$ (black line), 0.85 (blue line), 0.8 (red line). The density and the temperature have been considered at the pedestal-top $r / a \approx 0.9$. By moving along the vertical coordinate we find an increase of the fusion power of $200 \mathrm{MW}$ in correspondence of a variation of density of $\Delta n_{0} \approx 12 \%$. The trend is also evident on the temperature. By increasing the temperature of $\Delta T_{0} \approx 50 \%$ we observe an increment

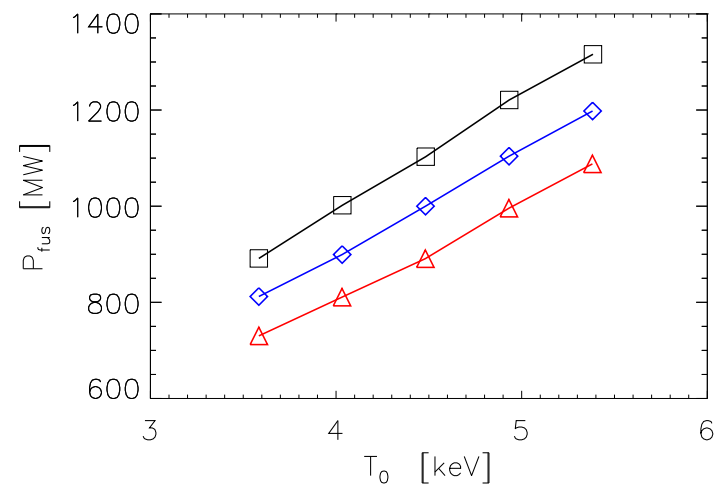

Figure 4. Fusion power as a function of temperature at the pedestal top. We have considered values of temperature and density at $r / a=0.9$. Three values of Greenwald fraction have been considered: $n_{0} / n_{G w}=0.9$ (black line), $n_{0} / n_{G w}=0.85$ (blue line), $n_{0} / n_{G w}=0.8$ (red line).

of the fusion power of $\Delta P_{f u s} \approx 380 \mathrm{MW}$. Thus, the impact of density $n_{0}$ on the fusion power production is stronger than that one of the temperature $T_{0}$. In Fig. 5 we show the $H$-factor as a function of the pressure $p_{c}=2 n_{0} k_{B} T_{0}$ (with $k_{B}$ Boltzmann constant) for the same values of density considered in Fig. 4. The $H$ factor is defined as the ratio between the global energy confinement time $\tau_{E}$ and the value of $\tau_{E}$ obtained via the $I P B 98(y, 2)$ scaling $[2,50]$ :

$H_{98}=\frac{\tau_{E}}{\tau_{E}^{I P B 98}}$

We observe a very weak dependence on the density for the $H$-factor. Moreover, we note that the $H$-factor is a function of the temperature. In particular we obtained $H_{98}=0.97$ for the nominal case in which we considered a pedestal-top temperature $T_{0}=5.5$ $\mathrm{keV}$. We would like to emphasize that the choice of the temperature value for the nominal case (around which we performed our scans) is consistent with the EPED stability calculation done in Ref. [51], thus representing a standard $\mathrm{H}$-mode with a pedestal top pressure consistent with the peeling-ballooning stability criterion. We recall that in this work we consider the temperature as a free parameter and in this way the regressions in the paper allows us to explore various regimes in a coherent manner. In this way, when we consider a pedestal model, we have a complete prediction of the stored energy of the plasma and the $\mathrm{H}$-factor becomes an output value. In case we do not have a pedestal model, we can use the $\mathrm{H}$ factor to deduce the characteristics of the pedestal top. The performed simulations for the case $B=5.8 \mathrm{~T}$ and $R=9 \mathrm{~m}$ show an $H$-factor lower than unity. 


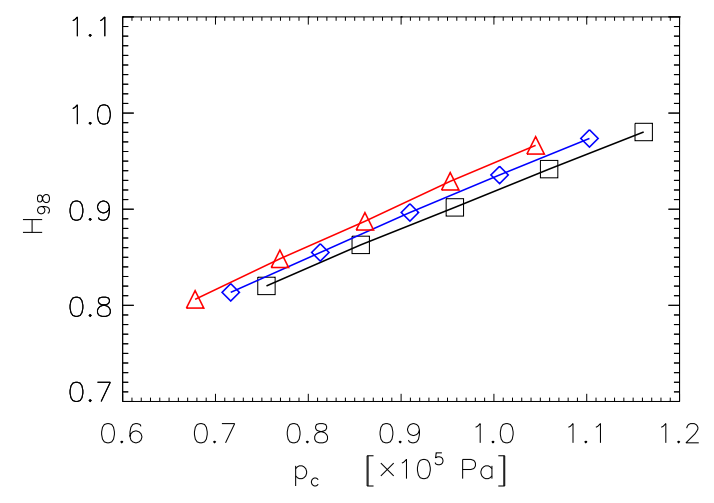

Figure 5. H-factor as a function of pressure calculate at the pedestal. In particular we have considered values of temperature and density at $r / a=0.9$. Three values of Greenwald fraction has been considered: $n_{0} / n_{G w}=0.9$ (black line), $n_{0} / n_{G w}=0.85$ (blue line), $n_{0} / n_{G w}=0.8$ (red line).

In Fig. 6 we show the fusion power as a function of pressure for several choices of parameters, whose combination delivers the same fusion power at the same edge pressure. In particular the green line describes a simulation obtained with $n_{0} / n_{G w}=0.9, B_{0}=5.8 \mathrm{~T}$ and $R_{0}=8.5 \mathrm{~m}$. The blue line has been obtained by considering $n_{0} / n_{G w}=0.85, B_{1}=B_{0}+7 \% B_{0}$, $R_{1}=R_{0}$ and the red line corresponds to the case $n_{0} / n_{G w}=0.8, B_{2}=B_{0}, R_{2}=R_{0}+7 \% R_{0}$. This can give important indications on the DEMO design on the basis of the technological and physical constraints. In fact, for a given pressure we can obtain a specific $P_{\text {fus }}$ value in several manner. We note that by increasing the magnetic field by a small amount, we can decrease the density and preserve the performance. The best conditions are obtained by increasing the radius by the same percentage as the magnetic field, further reducing the density at the pedestal top. This is related to the fact that, at fixed current, an increase of size of the tokamak implies a strong reduction of the density in the device. All these results can be quantified by means of scaling laws that relate the crucial quantities such as $P_{f u s}, H$-factor and the parameters investigated in the scans. In order to obtain the mentioned laws we adopt the well known least-squares method [52]. Thus, we find a relationship $y(x)=$ $y\left(x ; a_{j}\right)$ between measured dependent $y$-variables and independent $x$-variables with $a_{j}$ adjustable parameters. The latter are calculated by minimizing the quantity $\sum_{i=0}^{N}\left(y_{i}-y\left(x ; a_{j}\right)\right)^{2} / \sigma_{y}^{2}$ where $\sigma_{y}$ represents the standard deviation of the $y_{1} \ldots y_{N}$ measurements. In this work, we assume the following functional relationship $f=C_{0} \cdot C_{1}^{a_{1}} \ldots C_{M}^{a_{M}}$ between independent

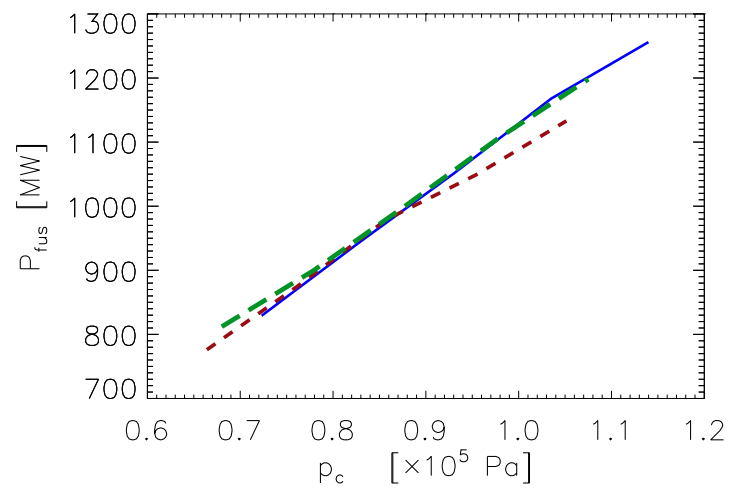

Figure 6. Fusion power as a function of pressure calculate at the pedestal for three different values of density. Continuous (blue) line corresponds to $R=8.5 \mathrm{~m}, B=$ 6.2 Tesla, $n_{0} / n_{G w}=0.85$, dash (red) line corresponds to $R=9 \mathrm{~m}, B=5.8 \mathrm{~T}, n_{0} / n_{G w}=0.8$, and dash-dotted (green) line corresponds to $R=8.5 \mathrm{~m}, B=5.8$ Tesla, $n_{0} / n_{G w}=0.9$.

Table 2. . Values of adjustable quantities $a_{j}$ and related errors for the different parameters in Eq. 8.

\begin{tabular}{l|llll}
\hline \multirow{4}{*}{$P_{\text {fus }}$} & $n_{0} / n_{G w}$ & $T_{0}$ & $R$ & $B$ \\
& +2.29 & +0.94 & -0.73 & +1.23 \\
& \pm 0.07 & \pm 0.02 & \pm 0.10 & \pm 0.09 \\
& & & & \\
& $e^{P_{a u x}}$ & $e^{P_{\text {rad }}}$ & $I_{p}$ & $A$ \\
+0.0040 & -0.00080 & +2.40 & +1.08 \\
& \pm 0.0001 & \pm 0.00002 & \pm 0.16 & \pm 0.19 \\
\hline
\end{tabular}

$f$-quantities (in our case $P_{\text {fus }}$ or $H_{98}$ ) and $C$ parameters of our scans and with a variable change we obtain a correspondence with a straight-line model. Thus, by considering all the performed simulations, for the fusion power $P_{\text {fus }}$ we obtain the following regression:

$$
\begin{aligned}
P_{\text {fus }}= & 0.053\left(\frac{n_{0}}{n_{G w}}\right)^{2.29} T_{0, e}^{0.94} R^{-0.73} \\
& B^{1.23} e^{0.004\left(P_{\text {aux }}-0.2 P_{\text {rad }}\right)} I_{p}^{2.4} A^{1.08}
\end{aligned}
$$

As expected, the exponent of the Greenwald fraction is around 2. The exponent of the temperature is approximately equal to 1 . This is due to the reduction of the temperature dependence of the cross section with the increasing temperature in the range between $\approx 30$ $\mathrm{keV}$ and $\approx 40 \mathrm{keV}$. Moreover, with the increase of the temperature, the profile stiffness becomes stronger. Concerning the major radius $R$, at the first impact, it could be surprising to find a negative exponent value. The inverse dependence of the major radius reflects the fact that by increasing $R$ the Greenwald density decreases and thereby at constant Greenwald fraction 
the density has to decrease at fixed current. The scaling law has been obtained by considering $C_{m}=$ $e^{P_{a u x}}$ and $C_{m+1}=e^{P_{\text {rad }}}$. Thus, also in the case in which $P_{\text {aux }}$ and $P_{\text {rad }}$ are equal to zero, the $P_{\text {fus }}$ must be different from zero. For this reason, we find $P_{a u x}$ and $P_{\text {rad }}$ as exponent in the $P_{\text {fus }}$ expression. We observe that $P_{\text {rad }}$ is multiplied by $c_{1}=0.2$. This small coefficient takes into account that $\mathcal{P}_{\text {rad }}$ is concentrated at the edge of the plasma, where we impose boundary conditions. Therefore $\mathcal{P}_{\text {rad }}$ cannot strongly influence the simulation results and in particular the physics of the core. Contrary, the effects of $\mathcal{P}_{\text {aux }}$ act along the radius and in particular in the core. Thus, the coefficient $c_{1}=0.2$ can be considered a number that recovers the relative importance between $P_{a u x}$ and $P_{\text {rad }}$. An important aspect is related to the accuracy with which parameters are determined by the data set of scans. In table 2 we show the exponent value for each parameter of our scan and the related errors for $P_{\text {fus }}$ in Eq. 8. Moreover, the standard deviation related to $P_{\text {fus }}$ is $\sigma_{P_{\text {fus }}}=23.5 \mathrm{MW}$. In a general way, all the exponents of scaling -except fusion power and density- are TGLF stiffness dependent. Therefore, in particular, the low influence of $P_{\text {rad }}$ on the physics of the core can be also, in part, related to the TGLF stiffness. In Fig. 7 the scaling law has been compared with the numerical results showing a good agreement between simulations and analytical expression. This agreement has been quantified through $\sigma_{P_{f u s}}$ and by means of the error values shown in table 2. In order to further verify the validity of Eq. 8 we performed a scan in a different range with respect to that one considered around the nominal case. The new scan in temperature and density has been performed with $B=5.26 \mathrm{~T}, q_{95}=3.5$ and $R=9,01 \mathrm{~m}$. In Fig. 8 we show that results from Eq. 8 (right lines) are able to well reproduce values obtained from simulations (triangles).

Eq. 8 that links $P_{\text {fus }}$ with $B, R, T_{0}, n_{0}, P_{\text {rad }}$, $P_{a u x}, I_{p}$ and $A$ can be very useful because it is written in engineering terms that are directly related to the practical design of control systems. However, often we can find basic discrepancies between engineering and plasma physics requirements, as the most performing plasma configuration may not be compatible with the different technological limits [53]. In the basic strategy of design analysis of the device, both points of view are important and for this reason by using Eq. 6 we write Eq. 8 as a function of the safety factor that, as discussed, represents a fundamental physical constraint:

$$
\begin{aligned}
P_{\text {fus }}= & 25\left(\frac{n_{0}}{n_{G w}}\right)^{2.29} T_{0, e}^{0.94} q_{95}^{-2.4} R^{1.67} A^{-3.7} \\
& B^{3.63} e^{0.004\left(P_{a u x}-0.2 P_{\text {rad }}\right)}
\end{aligned}
$$

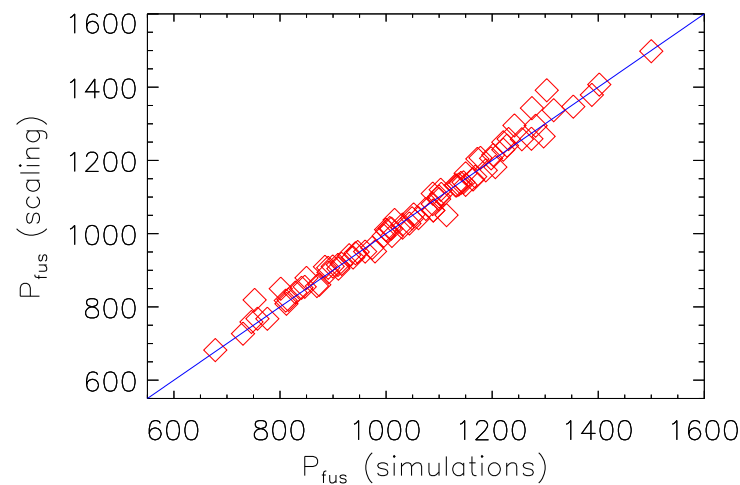

Figure 7. Fusion power of simulations v.s. fusion power obtained with the scaling law of Eq. 8 for the range of simulation values.

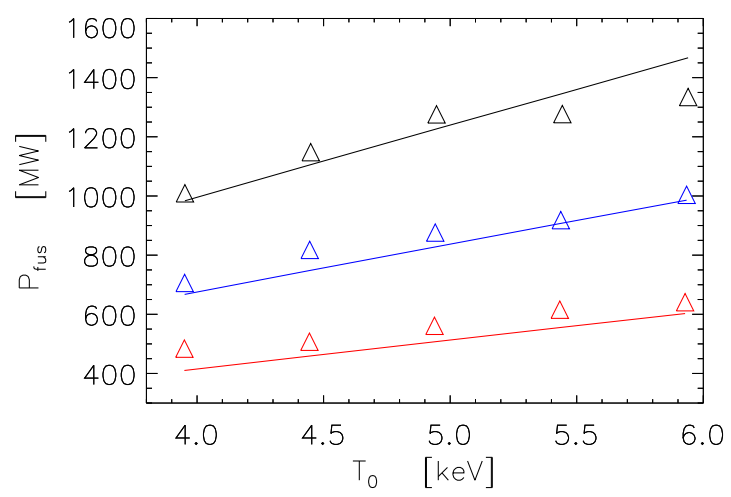

Figure 8. Comparison between scaling law prediction (see Eq. 8) and scan on temperature and density performed in a different DEMO regime with respect to the scan performed around the nominal case. The black, red and blue colors indicate $n_{0} / n_{G w}=0.6,0.75,0.9$ respectively.

This equation is perfectly consistent with Eq. 8. In this case, the positive exponent of the major radius reflects the fact that by increasing $R$, automatically the size of the machine increases and for a fixed value of the safety factor necessarily the current increases. The Greenwald limit decreases and consequently the plasma density decreases, but in lower extent with respect to the previous case. In order to discuss the consequence of this scaling law, we consider a device with a major radius $R=8.5 \mathrm{~m}$ and with a safety factor $q_{95} \approx 3.5$. In Fig. 9 for two values of the aspect ratio $A=2.7$ (top panel) and $A=3.1$ (bottom panel) we show the fusion power as a function of the pressure for $B=6.2 \mathrm{~T}$ (black line), $B=5.8 \mathrm{~T}$ (red line) and $B=5.4 \mathrm{~T}$ (yellow line) respectively. Each curve has 

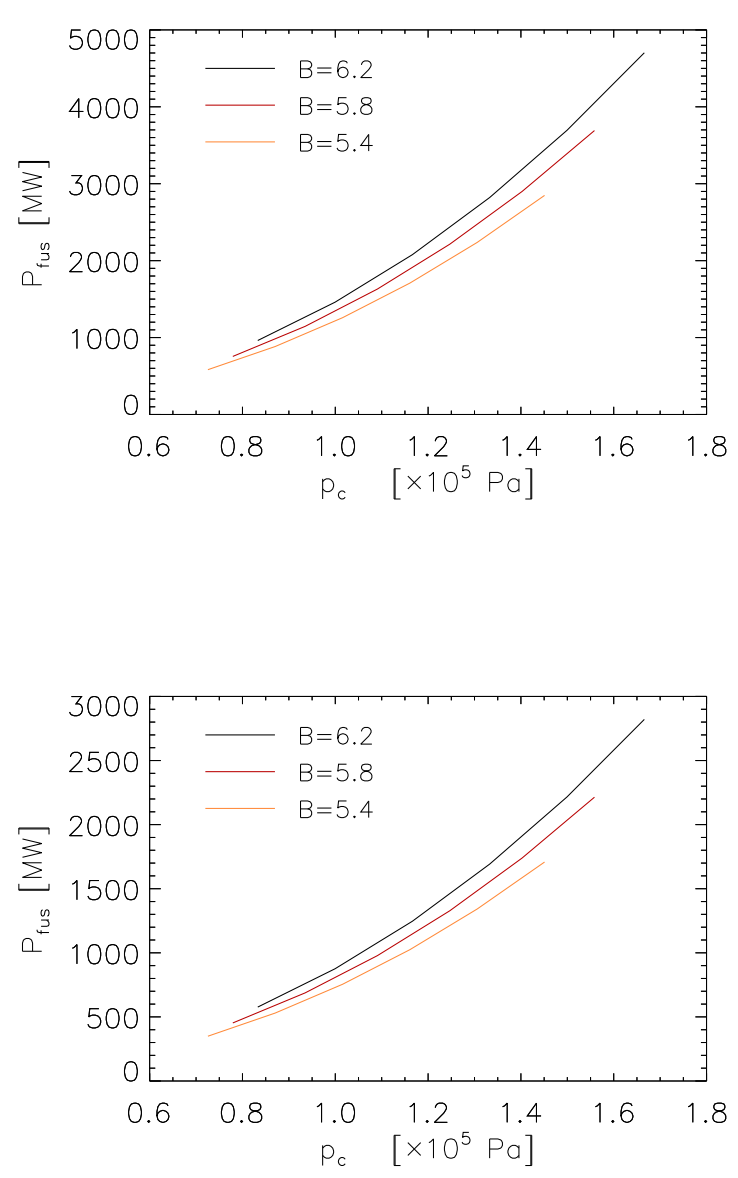

Figure 9. (Top panel) Fusion power as a function of pressure obtained with an aspect ratio $A=2.7$. (Bottom panel) Fusion power as a function of pressure obtained with an aspect ratio $A=3.1$.

been plotted by varying the density in the range $0.5 \leq$ $n_{0} / n_{G w} \leq 1$ at fixed pedestal-top temperature $T_{0}=6$ $\mathrm{keV}$. Already, for low values of the pressure we obtain very attractive values of the fusion power. For example, we consider the abscissa point $p_{c}=1.4 \cdot 10^{5} \mathrm{~Pa}$. By recalling that the temperature is fixed, we can associate to the pressure $p_{c}$, the density value $n_{0}=7.3 \cdot 10^{19}$ $\mathrm{m}^{-3}$. For $n_{0} / n_{G w}=0.84$ (black line), $n_{0} / n_{G w}=0.9$ (red line), $n_{0} / n_{G w}=0.96$ (yellow line) we obtain a fusion power around $2800 \mathrm{MW}$ and $1700 \mathrm{MW}$ for the case $A=2.7$ and $A=3.1$ respectively. We would like to emphasize that machines such as ITER have been designed to work in $H$-mode [54]. Also DEMO is planned to work in $H$-mode, but problems linked to ELMs are not acceptable. For this reasons, it can be very useful to investigate new regimes in which DEMO could operate.

A very promising regime is represented by the $I$ - mode as an example of regime where the boundary of the pedestal stability is not reached. This regime presents comparable values of temperature of the $H$ mode at the pedestal and it is characterized by lower values of density. However, due to different conditions in which several tokamak machine operate it is not clear the exact pressure characteristics by which $I$ mode can be identified. In order to establish a regime in which we can avoid the problem of ELMs, it is important to identify the pressure conditions in which $I$-mode exist. To this purpose can be useful to write Eq. 9 in terms of the pressure $p_{c, e}$ :

$$
\begin{aligned}
P_{\text {fus }}= & 6.5\left(\frac{n_{0}}{n_{G w}}\right)^{1.35} p_{c, e}^{0.94} q_{95}^{-1.46} R^{2.61} A^{-3.7} \\
& B^{2.69} e^{0.004\left(P_{\text {aux }}-0.2 P_{\text {rad }}\right)}
\end{aligned}
$$

In Eq. 10 the pressure $p_{c, e}=n_{0} T_{0, e}$ is calculated by considering $n_{0}$ normalized to $10^{20} \mathrm{~m}^{-3}$ and by expressing electron temperature $T_{0, e}$ at the pedestal top in $\mathrm{keV}$. In order to investigate the potentialities of the $I$-mode regime, we use Eq. 10 and a simple model based on the experimental results observed in ASDEX Upgrade [55]. It has been reported [56] that $H$-mode is characterized by a parameter $\eta=\left(1 / L_{T}\right) /\left(1 / L_{n}\right)=$ $(d \ln T / d r) /(d \ln n / d r)$ in the following range $0.5 \lesssim$ $\eta_{H} \lesssim 1$. The $I$-mode presents values in the range $2.5 \lesssim \eta_{I} \lesssim 5$ so with an $\eta_{I} \approx 5 \eta_{H}$. A similar relation between $\eta_{I}$ and $\eta_{H}$ has been obtained by means of experiments on Alcator C-Mod [15]. On the basis of these considerations we model the pedestal profile of temperature and density used in our simulations with the following expressions:

$$
\begin{aligned}
& T=\frac{1}{2}\left[2 T_{0}-\Delta T\left(1+\tanh \left(\frac{x-x_{1}}{l_{T}}\right)\right]\right. \\
& n=\frac{1}{2}\left[2 n_{0}-\Delta n\left(1+\tanh \left(\frac{x-x_{1}}{l_{n}}\right)\right]\right.
\end{aligned}
$$

in the region $0.90 \lesssim x \lesssim 1$ with $x=r / a$. In order to reproduce the pedestal conditions adopted in the simulations we consider a temperature value $T_{0}=5.5 \mathrm{keV}$ and a density value $n_{0}=0.9 n_{G w}$ around $r / a=0.92$. By considering a magnetic field $B=6.2 \mathrm{~T}$, a safety factor $q_{95}=3.5$ and a major radius $R=9 \mathrm{~m}$ we obtain $n_{G w}=8.18 \cdot 10^{19} \mathrm{~m}^{-3}$. Moreover, by considering the width of pedestal region we impose $l_{T}=l_{n}=0.015$ and we choose $\Delta T=5.5 \mathrm{keV}$ and $\Delta n=0.5 n_{G w}$ to obtain $T \approx 0$ and $n=0.4 n_{G w}$ at $r / a=0.98$. These profiles well represent attempt $H$-mode conditions at the pedestal in DEMO. We discover in this way that the ratio $\eta_{H}$ calculated around $x_{1}=0.95$ will be larger than the $\eta_{H}$ observed in ASDEX Upgrade. In particular we obtain for an $H$ mode $\eta_{H} \approx 3.3$ in DEMO. 
Starting from this representation we impose for an $I$-mode regime the same profile of temperature modeled for the $H$-mode and we consider $\eta_{I} \approx 4 \eta_{H} \approx$ 13.2. The density at the pedestal in this case decreases at the value $n_{0} \approx 0.75 n_{G w} \approx 6.14 \cdot 10^{19} \mathrm{~m}^{-3}$ and the pressure is $p_{c, e} \approx 0.54 \cdot 10^{5} \mathrm{~Pa}$. By maintaining fixed the pressure and by varying the density, on the basis of Eq. 10, we investigate the fusion power behavior. In Fig. 10 (top panel) in particular we show the fusion power as a function of $n_{0} / n_{G w}$ for several values of the magnetic field. We note that by maintaining fix the safety factor $q_{95}$ and the major radius $R$, for each values of the magnetic field we have a different value of the current. For this reason, in correspondence to several $I_{p}$ values we have different Greenwald limits and different temperatures. In Fig. 10 (bottom panel) we show the temperature as a function of $n_{0} / n_{G w}$ by maintaining the pressure constant. We observe that with $n_{0} / n_{G w}=0.75$ and $T_{0}=5.5 \mathrm{keV}$ for $B=6.2 \mathrm{~T}$ and $R=9 \mathrm{~m}$, we can have a fusion power $P_{\text {fus }}=1400 \mathrm{MW}$. If we consider that for this case $P_{\text {aux }}=50 \mathrm{MW}$ we find $Q=P_{\text {fus }} / P_{\text {aux }} \approx 28$ that is very promising for a realistic DEMO design. Similar results can be obtained for a different radius $R$ of the machine by fixing the aspect ratio $A$. To this purpose, in Fig. 11 we show the fusion power and the temperature as a function of the Greenwald fraction. In Fig. 12 we compare two representative equilibrium profiles in stationary phase for $H$-mode and $I$-mode regimes by choosing pedestal conditions on the basis of previous considerations.

To give a complete picture of the DEMO operation in different regimes another important parameter, useful to characterize the performance of the machine, is the energy confinement time $\tau_{E}$. This parameter with $P_{\text {fus }}$ and $Q$ is involved in the relation of the thermal equilibrium of the tokamak plasma:

$\left(f_{\text {coup }}+\frac{1}{Q}\right) P_{\text {fus }}=\frac{W_{t}}{\tau_{E}}$

where $W_{t}=W_{e}+W_{i}$ is the total thermal energy of the plasma. As we previously discussed, the confinement time can be expressed via the $H$-factor that compares $\tau_{E}$ with the standard confinement time of the scaling law $\tau_{E}^{I P B 98}$. Thus, by means of our scans we obtain the following scaling law for the $H$-factor:

$$
\begin{aligned}
H_{98}= & 0.33\left(\frac{n_{0}}{n_{G w}}\right)^{-0.13} p_{c, e}^{0.49} R^{-0.27} \\
& B^{-1.21} e^{-0.0003\left(P_{\alpha}+P_{\text {aux }}+P_{\text {rad }}\right)} q^{1.30} A^{1.41}
\end{aligned}
$$

In this way we are able to associate to the fusion power the corresponding $H_{98}$ value. In Fig. 13 the scaling law of Eq. 14 has been compared with the numerical results showing a very good agreement between simulations and the $H_{98}$ expression. This agreement can be quantified via the standard deviation
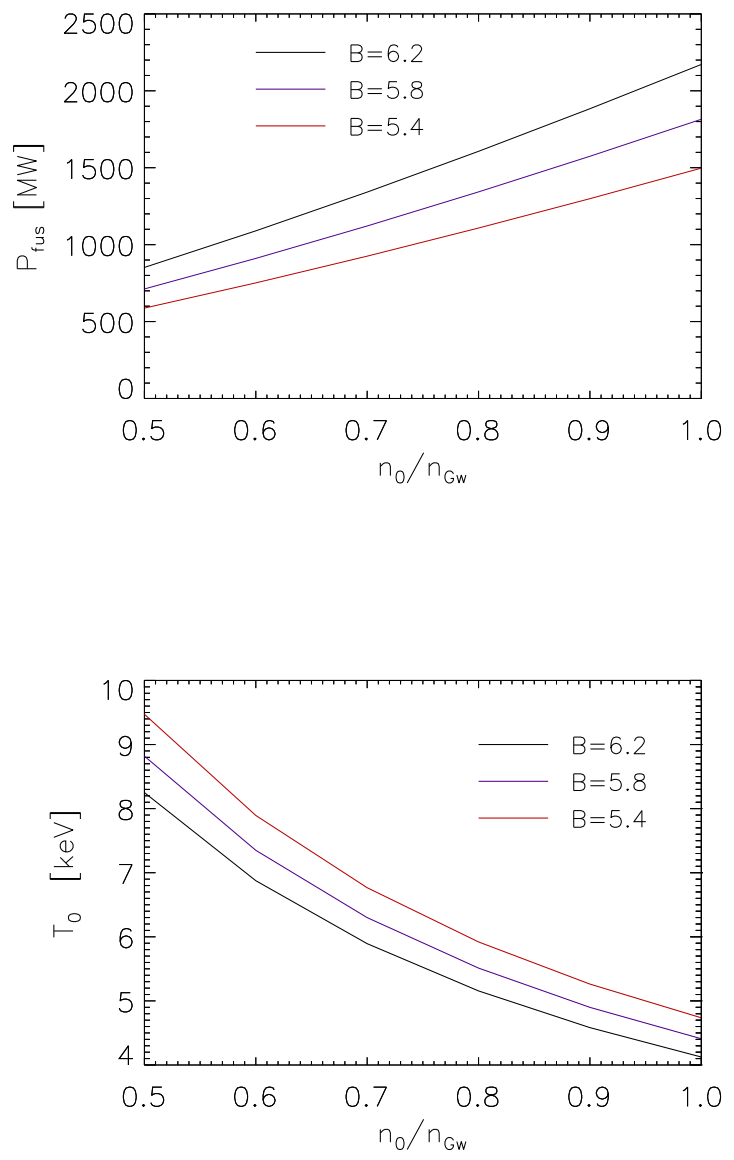

Figure 10. (Top panel) Fusion power in I-mode as a function of Greenwald fraction for several value of magnetic field. The plot has been obtained with a fixed value $p_{c}$ of the kinetic pressure. (Bottom panel) Pedestal top temperature $T_{0}$ as a function of the Greenwald fraction for several values of the magnetic field. The curves have been calculated by maintaining the pressure constant at a fixed value $p_{c}$.

Table 3. Values of adjustable quantities $a_{j}$ and related errors for the different parameters in Eq. 14.

\begin{tabular}{l|llll}
\hline \multirow{4}{*}{$H_{98}$} & $n_{0} / n_{G w}$ & $p_{c, e}$ & $R$ & $B$ \\
& -0.13 & +0.49 & -0.27 & -1.21 \\
\pm 0.02 & \pm 0.01 & \pm 0.07 & \pm 0.07 \\
& & & & \\
& $e^{P_{\alpha}+P_{\text {aux }}}$ & $e^{P_{\text {rad }}}$ & $q$ & $A$ \\
-0.00030 & -0.00030 & +1.30 & +1.41 \\
& \pm 0.00002 & \pm 0.00002 & \pm 0.07 & \pm 0.16 \\
\hline
\end{tabular}

value that for $H_{98}$ is $\sigma_{H_{98}}=0.01$ and through the errors for the different parameters of Eq. 14 shown in table 3 .

By using the nominal values of several machines, Eq. 14 allows us to obtain the expected $H$-factor 

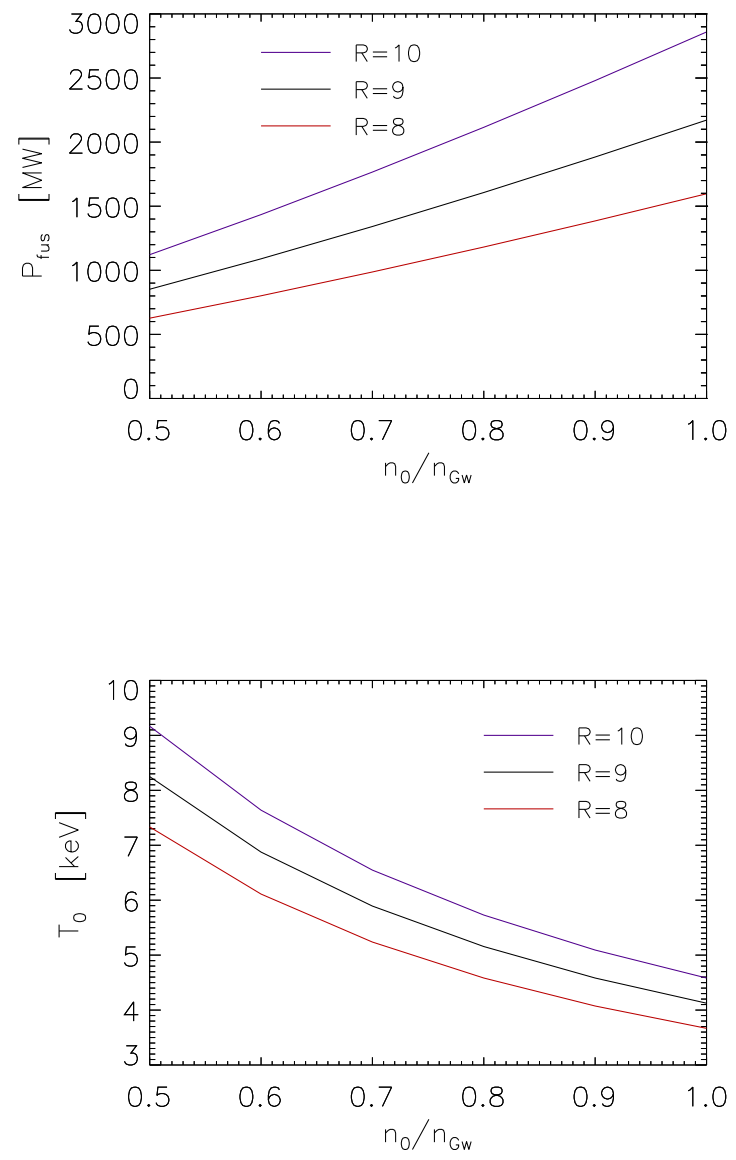

Figure 11. (Top panel) Fusion power as a function of Greenwald fraction for several value of major radius. The plot has been obtained with a fixed value $p_{c}$ of the kinetic pressure. (Bottom panel) Pedestal top temperature $T_{0}$ as a function of the Greenwald fraction for several values of the major radius. The curves have been calculated by maintaining the pressure constant at a fixed value $p_{c}$.

value for devices such as ARC, DEMO and also ITER. However, with this scaling law we cannot obtain the correct $H$-factor value of ASDEX Upgrade and smaller machines. This is principally due to dependence of $P_{f u s}$ - and consequently of $P_{\alpha}$ - on the size of the machines. Essentially, our scaling law cannot be applied to devices having zero or too small values of $P_{\text {fus }}$. Starting from Eq. 14, by eliminating the temperature we can also write $P_{\text {fus }}$ as a function of $H_{98}$ :

$$
\begin{aligned}
P_{\text {fus }}= & 50.7\left(\frac{n_{0}}{n_{\text {Gw }}}\right)^{1.81} H_{98}^{2.15} R^{3.55} \\
& B^{5.64} e^{0.0052 P_{\text {aux }}} e^{-0.00015 P_{\text {rad }}} q^{-4.43} A^{-7.4}
\end{aligned}
$$

The expression of $P_{\text {fus }}$ takes into account the implicit dependence of the temperature from the size of the
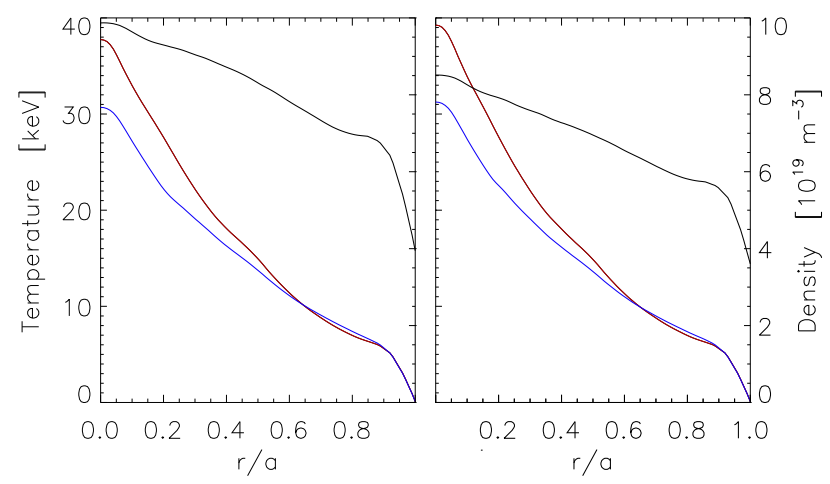

Figure 12. Example of equilibrium profiles for $H$-mode (left) and I-mode (right) obtained with ASTRA. Left and right axis indicate temperature and density respectively with the same scale for both the figures. Ion and electron temperature profiles are indicated with blue and red lines respectively, while density profiles are indicated with black lines.

tokamak. In Fig. 14 we show the fusion power as a function of the dimension of the machine for $\mathrm{H}$ mode and $I$-mode and - for a sake of completeness we also discuss the $L$-mode regime. The quantities in common to these three regimes considered for the plot are $B=6 \mathrm{~T}, q_{95}=3.5, A=3, P_{\text {aux }}=50 \mathrm{MW}$ and $P_{\text {rad }}=200 \mathrm{MW}$. It is important to note that Eq. 15 is related to the constraint $P_{\text {fus }} / 5+P_{\text {aux }}-P_{\text {rad }}>0$. Starting from the considered values of $P_{a u x}$ and $P_{\text {rad }}$, we have $P_{f u s}>750 \mathrm{MW}$. For the $H$-mode we consider a $H$-factor and a Greenwald fraction close to 1 and we obtain $P_{f u s}=2300 \mathrm{MW}$ with a $Q=46$ in correspondence of $R=8.5 \mathrm{~m}$. For the same machine the $I$-mode with $n_{0} / n_{G w}=0.75$ presents a $P_{f u s} \approx$ 1400 with a $Q \approx 28$. The value $P_{\text {fus }}=2000 \mathrm{MW}$ is reached in $I$-mode at $R=9.5 \mathrm{~m}$. However, we note that due to the strong dependence of $P_{f u s}$ from the aspect ratio, by decreasing the $A$ parameter, we can further increase the performance of the $I$-mode regime. In correspondence of $R=8.5 \mathrm{~m}$ the $L$-mode shows $P_{\text {fus }}=400 \mathrm{MW}$ with a low value of $Q$. Moreover, the dimension of a tokamak that operates in $L$-mode with $P_{\text {fus }}=2000 \mathrm{MW}$ is around $R=13 \mathrm{~m}$. These values are summarized in Table 4.

\section{Conclusion}

Because of the complexity of heat and particle transport processes in tokamak plasmas, it is challenging to accurately predict a burning plasma scenario. For this reason, the description of the global energy by scaling 
Table 4. DEMO performance in different regimes for $R=8.5 \mathrm{~m}$, $B=6 \mathrm{~T}, q_{95}=3.5, A=3$.

\begin{tabular}{lllll}
\hline Regime & $n_{0} / n_{G w}$ & $P_{\text {fus }}[\mathrm{MW}]$ & $Q$ & $H_{98}$ \\
\hline$H$-mode & 0.90 & 2300 & 46 & 0.9 \\
\hline$I$-mode & 0.75 & 1400 & 28 & 0.8 \\
\hline$L$-mode & 0.75 & 400.0 & 8 & 0.5 \\
\hline
\end{tabular}

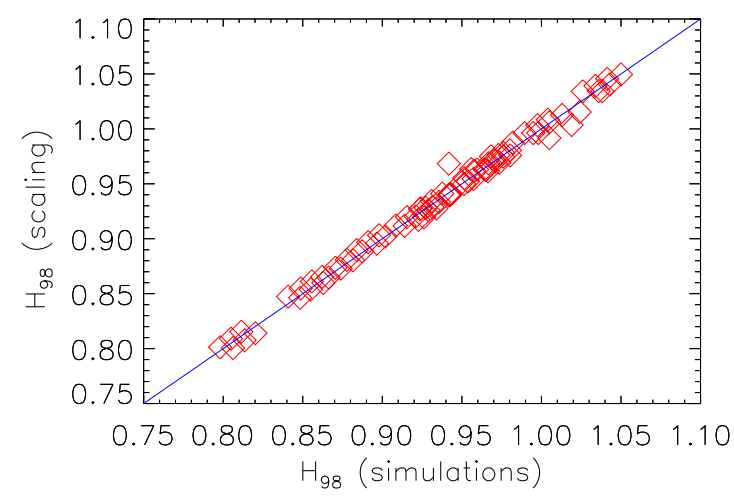

Figure 13. H-factor of simulations v.s. H-factor obtained with the scaling law of Eq. 14 for the range of simulation values.

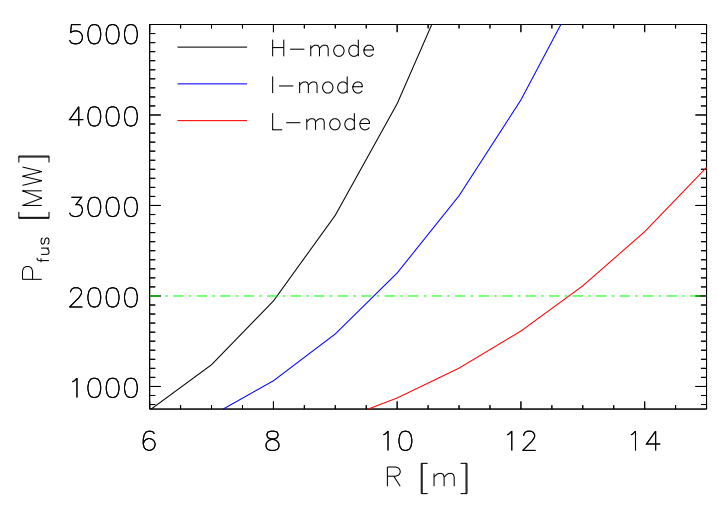

Figure 14. Fusion power as a function of size of the machine for $H$-, $I$-, L-mode. The quantities in common to the regimes considered for the plot are $B=6 T, q_{95}=3.5$, $A=3, P_{a u x}=50 \mathrm{MW}$ and $P_{\text {rad }}=200 \mathrm{MW}$. Starting from these values and in agreement with the constraint $P_{\text {fus }} / 5+P_{\text {aux }}-P_{\text {rad }}>0$ the minimum value of the fusion power that can be plotted is $P_{f u s}=750 \mathrm{MW}$. laws operating in regimes such as $H$-mode represents a key tool in extrapolating plasma performance in tokamaks. In this work we used the 1.5 dimensional transport ASTRA code and the TGLF model to deduce scaling laws for fusion power and $H$-factor in several regimes of interest for DEMO scenarios. In particular, with the ASTRA code we performed several scans on different parameters in order to investigate the performance of the device in a large range of values. Starting from a nominal case, by varying one quantity at the time and by maintaining fix the others, we performed scans in geometrical parameters such as major radius and aspect ratio, in external parameters such as magnetic field, current, radiation and auxiliary power and in pedestal parameters such as temperature and density at the pedestal top. Particular attention has been made for the latter. The pressure conditions can help to distinguish between the different regimes. By means of a simple model, based on temperature and density gradient, we describe the performance of DEMO by distinguishing between the different regimes. We know that $H$-mode is one of the most robust regimes able to improve tokamak confinement, but the presence of ELMs is not acceptable in DEMO. For this reason, in parallel to the $H$-mode, it has been interesting to investigated new scenarios in other regimes such as the $I$ mode or regimes in which the boundary of the pedestal stability is not reached. This regime is characterized by a temperature pedestal similar to $H$-mode without a density pedestal and it has the advantage over the ELMy $H$-mode of eliminate transient heat flux pulses to divertor. The main result obtained for the different scenarios considered in this paper is linked to the performance of DEMO. In this work we show that it is possible to move towards an $I$-mode avoiding the problems of ELMs, obtaining at the same time very good performance for DEMO in terms of the fusion power, $H$-factor and $Q$. Finally, we would like to emphasize that we investigate baseline regimes without the inclusion of important effects like nonlinear zonal flow physics $[57,58]$, inner transport barrier and so on. These latter can improve the confinement and in principle they point to confirm and to strengthen the obtained results. These aspects will be object of a future work.

\section{Acknowledgments}

This work has been carried out within the framework of the EUROfusion Consortium and has received funding from the Euratom research and training programme 2014-2018 and 2019-2020 under grant agreement No 633053. The views and opinions expressed herein do not necessarily reflect those of the European Commission. 


\section{References}

[1] Becoulet A. 2013 Nucl. Fusion 53104023

[2] ITER Physics Expert Groups on Confinement, Transport, Confinement Modeling and Database, ITER Physics basis editors 1999 Chapter 2: Plasma confinement and transport Nucl. Fusion 392175

[3] ITER www.iter.org/

[4] Federici G. et al. 2017 Nucl. Fusion 57092002

[5] Zohm H. 2010 Fusion Sci. Technol. 58613

[6] EUROfusion www.euro-fusion.org/glossary/tokamak/

[7] Zohm H. et al. $2017 \mathrm{Nucl}$. Fusion 57086002

8] Federici G. et al. 2018 Fusion Eng. Design 136729

[9] Pereverzev G.V. et al. 1991 ASTRA an automated system for transport analysis, IPP Report 5/42, Max-PlanckInstitut fuer Plasmaphysik

[10] Staebler G.M. et al. 2005 Phys. Plasmas 12102508

[11] Wagner F., 1984 Phys. Rev. Lett. 531453

[12] Ryter F. et al. 1998 Plasma Phys. Control. Fusion 40725

[13] Loarte A. et al. 2003 Plasma Phys. Control. Fusion 451549

[14] Sips A. C. C. et al. 2005 Plasma Phys. Control. Fusion 47 A19

[15] Whyte D. G. et al. 2010 Nucl.Fusion 50105005

[16] Hubbard A. E. et al. 2016 Nucl. Fusion 56086003

[17] Hubbard A. E. 2017 Nucl. Fusion 57126039

[18] Cziegler I. et al., 2013 Phys. Plasmas 20055904

[19] Ryter F. et al. 2017 Nucl. Fusion 57016004

[20] Fable E. et al 2010 Plasma Phys. Control. Fusion 52015007

[21] Fable E. et al 2013 Plasma Phys. Control. Fusion 55074007

[22] Siccinio M. et al. 2018 Nucl. Fusion 58016032

[23] Wesson J. 2004 Tokamaks Clarendon Press-Oxford

[24] Trubnikov B. A. 1979 Review of Plasma Physics, Ed. M.A Leontovich, Vol. 7, New York, p. 345

[25] Post D. E. et al. 1977 Atomic Data and Nuclear Data Tables 20397

[26] Sommer F. et al. 2012 Nuclear Fusion 52114018

[27] Creely A. J. et al. 2017 Phys. Plasmas 24056104

[28] Fable E. et al. Nuclear Fusion (submitted)

[29] Ivanov et al. 2005 32nd EPS Conf. on Plasma Physics 29 (ECA) P-5.063

[30] Houlberg W. A. et al. 1997 Phys. Plasmas 43230

[31] Staebler G. M. et al. 2005 Phys. Plasmas 12102508

[32] Staebler G. M. et al. 2007 Phys. Plasmas 14055909

[33] Kinsey J. E. et al. 2008 Phys. Plasmas 15055908

[34] Waltz R. E. et al. 1997 Phys. Plasmas 42482

[35] Staebler G. M. et al., 2013 Phys. Rev. Let. 110055003

[36] Candy J. et al. 2003 Comput. Phys. 186545

[37] Kallenbach A. et al. 2015 Nucl. Fusion 55053026

[38] Rhodes T.L. et al. 2011 Nucl. Fusion 51063022

[39] Sommer F. et al. 2015 Nucl. Fusion 55033006

[40] Erofeev I. et al. 2017 Nucl. Fusion 57126067

[41] Baiocchi B. et al. 2015 Plasma Phys. Control. Fusion 57 035003

[42] McClenaghan J. et al. 2017 Nucl. Fusion 57116019

[43] C. Pan et al. 2017 Nucl. Fusion 57036018

[44] McMillan B. F. et al. 2010 Phys. Rev. Let. 105155001

[45] Greenwald M. 2002 Plasma Phys. Control. Fusion 44 R27R 80

[46] Saibene G. et al. 1999 Nucl. Control. Fusion 40851

[47] Lang P.T. et al 2014 Nucl. Fusion 54083009

[48] Osborne T. H. et al. 2001 Phys. Plasmas 82017

[49] Stober J. et al. 2001 Nucl. Fusion 411535

[50] Uckan N.A. 1989 ITER physics design guidelines, ITER TN-PH-8-6

[51] Saarelma S. et al. 2018 Plasma Phys. Control. Fusion 60 014042

[52] Press W. H. et al. 1988 Numerical Recipes In C, Cambridge University Press

[53] Duchateau J.-L. et al. 2014 Fusion Eng. Des. 892606

[54] Hender T.C. et al. 2007 Progress in the ITER Physics
Basis: Chapter 3. MHD stability, operational limits and disruptions Nucl. Fusion 47 S128202

[55] Neuhauser J. et al 2002 Plasma Phys. Control. Fusion 44 855

[56] Palermo F. et al. 2016 Europhys. Lett. 115, 15001

[57] Palermo F. et al. 2015 Phys. Plasmas 22042304

[58] Ghizzo A. and Palermo F., 2015 Phys. Plasmas 22082303 